This item was submitted to Loughborough's Research Repository by the author.

Items in Figshare are protected by copyright, with all rights reserved, unless otherwise indicated.

\title{
The use of aerial images and GPS for Mobile Robot Waypoint Navigation
}

PLEASE CITE THE PUBLISHED VERSION

http://dx.doi.org/10.1109/TMECH.2008.2005197

PUBLISHER

(c) IEEE

VERSION

AM (Accepted Manuscript)

LICENCE

CC BY-NC-ND 4.0

REPOSITORY RECORD

Shair, Sultan, Jim H. Chandler, Victor J. Gonzalez Villela, Robert M. Parkin, and Michael R. Jackson. 2019. "The Use of Aerial Images and GPS for Mobile Robot Waypoint Navigation". figshare.

https://hdl.handle.net/2134/14831. 
This item was submitted to Loughborough's Institutional Repository (https://dspace.lboro.ac.uk/) by the author and is made available under the following Creative Commons Licence conditions.

\section{creative
commons}

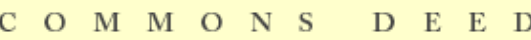

Attribution-NonCommercial-NoDerivs 2.5

You are free:

- to copy, distribute, display, and perform the work

Under the following conditions:

Attribution. You must attribute the work in the manner specified b the author or licensor.

Noncommercial. You may not use this work for commercial purposes.

No Derivative Works. You may not alter, transform, or build upon this work.

- For any reuse or distribution, you must make clear to others the license terms of this work.

- Any of these conditions can be waived if you get permission from the copyright holder.

Your fair use and other rights are in no way affected by the above.

This is a human-readable summary of the Leqal Code (the full license).

\section{Disclaimer 만}

For the full text of this licence, please go to: http://creativecommons.org/licenses/by-nc-nd/2.5/ 


\title{
The Use of Aerial Images and GPS for Mobile Robot Waypoint Navigation
}

\author{
S. Shair, J. H. Chandler, V. J. Gonzalez-Villela, R.M. Parkin, Member, IEEE, and M.R. Jackson
}

\begin{abstract}
The application of aerial and satellite imagery for mobile robot path planning and navigation has shown potential in recent years. These uses vary from identifying terrain properties for creating traversability maps to extracting landmarks for autonomous navigation. With the freely available differential positioning system WAAS/EGNOS, the use of the Global Positioning System with aerial images providing valuable contextual data, demonstrates potential in waypoint-based navigation of mobile robots. However, important issues relating to the spatial accuracies of image, waypoint and GPS derived data, vital for obtaining accurate navigation results, are often overseen. This paper defines the causes of spatial inaccuracies in order to develop optimal waypoint navigation parameters and provides researchers with sufficient knowledge to reproduce similar results. An improvement of up to $48 \%$ in the number of waypoints reached, depending on the radius, was determined for the positional correction of the GPS. The reader is presented with a method for easily creating waypoints from aerial images, yielding results to a similar level of accuracy to conventional and often tedious manual methods. The results are shown with a simulated synchronous drive robot in Matlab's Simulink ${ }^{\circledR}$ environment.
\end{abstract}

Index Terms - Aerial imagery, GPS, Map Datum, Mobile Robot, Synchronous Drive Robot, Orthorectification, Overhead Images, WAAS, EGNOS, and Waypoints.

\section{INTRODUCTION}

The use of aerial and satellite imagery for agricultural and horticultural applications such as precision farming and for long-range autonomous terrain navigation has been a strong motivation behind research conducted at Loughborough University [1], in which a grass-cutting mower, the Ransomes Spider $^{\circledR}$, has been refitted for autonomous navigation (Fig. 1). In recent years there has been growing interest in imagery (aerial, satellite, LADAR/LIDAR, DEM and more) for robot

Manuscript received December 10, 2007. This work is supported by the Engineering and Physical Sciences Research Council (EPSRC), UK, and the Innovative Manufacturing and Construction Research Centre (IMCRC), Loughborough University, UK.

S. Shair, R. M. Parkin, and M. R. Jackson are with the Mechatronics Research Centre, Holywell Building, Loughborough University, Loughborough, LE11 3UZ, UK (phone: + 44 (0) 150922 6574; fax: + 44 (0) 150922 6577; e-mail: S.Shair@lboro.ac.uk).

J. Chandler is with the Department of Civil Engineering at Loughborough University, UK (e-mail: J.H.Chandler@lboro.ac.uk).

V. J. Gonzalez-Villela is with the Department of Mechatronics Engineering, UNAM, Mexico City, Mexico (e-mail: vjgv@servidor.unam.mx).

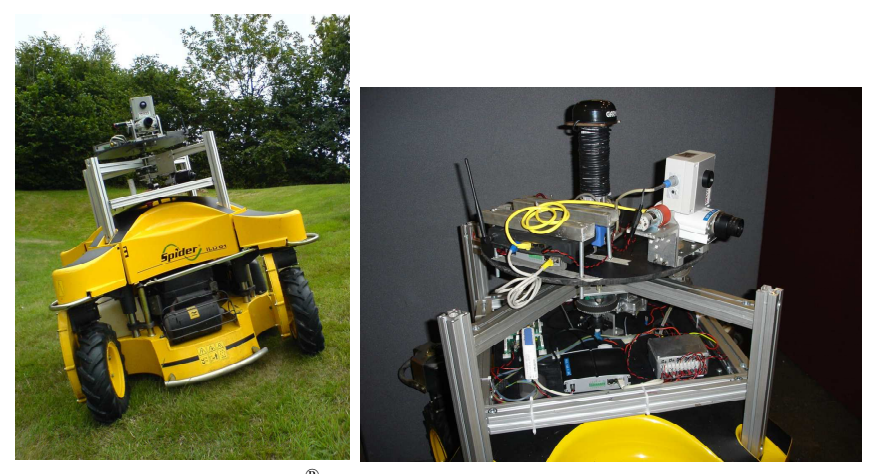

Fig. 1. Spider ${ }^{\circledR}$, refitted for autonomous navigation

path planning [2]-[9]. Each of these research activities addresses path planning and navigation differently. Researchers need to comprehend a range of complex issues involved in navigation, even though these may not be directly related to their prime research. One such task is the process of collecting a series of waypoints for mobile robot path planning. The most common procedure is through manual collection (surveying) of a series of waypoints using a high precision differential GPS receiver [4], [10], either using realtime (RTK) or post-processing the data. Even though this is a simple task, it is time consuming and requires thorough knowledge of the robot's working environment.

The concept of using imagery for defining waypoints is not a new idea. Freely available GIS tools such as Google Earth are often used by civilians in order to define their own route of travel [11], whether it be for hiking or driving. For in-car GPS navigation, the accuracy of these points is not of great importance since the waypoints are often conveyed relative to a global fixed street network and are not required for autonomous navigation - therefore positional inaccuracies from the GPS receiver and the waypoint positional resolution do not act as a major hindrance on the system's overall performance. However, for applications requiring higher navigation precision and autonomy, such as in mobile robots, greater significance must be attributed to image settings and coordinate reference systems to improve the waypoint accuracy, and GPS settings to ensure that the waypoints are reached.

The freely available differential GPS (WAAS/EGNOS) system shows potential for mobile robots as it offers positional accuracy to within three meters. It can be used in conjunction with aerial images for mobile robot waypoint navigation and is an exciting area of development. The upcoming deployment of 
the Galileo system (Europe's alternative to the GPS, which promises positional accuracy to within one meter with no signal degredation all year round [12]) potentially paves the way for replacing inertial measurement units (IMUs) coupled with satellite positioning. This will inevitably allow for the combination of aerial imagery and satellite positioning as a stand-alone application for mobile robot localisation only.

This paper is divided into four main sections. The first, entitled Waypoint Navigation, will discuss recent work which uses waypoints in mobile robot path planning. That will then lead the reader into the current uses of waypoint determination using imagery. The second section, Imagery, will discuss the relative advantages and disadvantages of aerial over satellite imagery, the critical process of georeferencing (i.e. calibration) of these images and finally the selection of the correct map settings (map datum and projection) for the working area. Both steps are critical if direct spatial comparison is desired.

The third section, Robotic System, will briefly discuss the Global Positioning System, and will provide the reader with a novel derivation of the kinematic model for a synchronous drive robot and introduce the controller used for the simulation. The fourth, entitled Experimental Results, will demonstrate the effect of GPS positional correction, accuracy and repeatability on waypoints reached. It will also demonstrate the waypoint accuracy that can be typically obtained using an orthorectified aerial image. Finally, a simulation of waypoints reached using a synchronous drive robot will be presented.

\section{WAYPOINT NAVIGATION}

In mobile robots it is quite difficult to separate the concepts of path planning and navigation, since a path is often planned with an appropriate controller in mind. A vast number of path planning techniques are in existence and in this paper a deliberative approach is used, wherein the robot follows a predefined trajectory, or a series of points.

Reference [13] terms pre-defined path planning as the "railway track algorithm" because the vehicle is confined to specific paths or roadways (the "tracks"). This is usually done when the coordinates of the path to be traversed are given to the robot in a series of known coordinates (waypoints). Classical path planning techniques assume a full knowledge of the robot's environment, which is believed to be correct and complete, but since complete knowledge of the environment for outdoor robots is not possible, a method employing waypoint-type algorithms is suitable [14].

It has been shown that a low-cost educational robot equipped with only a GPS receiver as its sensor, has obtained good results for waypoint navigation [15]. This method was also implemented on a boat yielding similar results. Waypoint navigation has also been used in an autonomous boat, yielding satisfactory results through the use of differential GPS [16]. With positional fix updates roughly once per second, the author was able to achieve positional accuracy up to one meter for the application. Furthermore, work conducted on the use of waypoints for an autonomous kiteplane, implementing lowcost sensors, achieved successful maneuvering under wind disturbances [17].

The use of waypoints derived from aerial imagery has also been well received by some researchers working on mobile robots in rugged outdoor environments [18], [19].

In summary, the use of waypoints and GPS have been shown to be powerful tools for outdoor mobile robot navigation. For this research, waypoints will refer to outdoor points within a predefined positional coordinate system, which will be clarified in the following sections.

\section{IMAGERY}

\section{A. Aerial Images}

As mentioned previously, a recent trend in navigation and area representation methods has been the use of various types of imagery. This work will focus on the use of aerial images (photographs) and not 3D Digital Elevation Models (DEM) such as LIDAR/LADAR, since low-cost GPS units do not provide accurate altitude data. Freely available or low-cost imagery (e.g. Google Earth) can be several years old and of variable image resolution, rendering it useless for many applications; yet, freely available data remains useful for conveying the landscape for various purposes.

There are many types of orbital satellites which collect images, such as Landsat, SPOT and IRS; however, most have a lower resolution (i.e. less detail) than the recently-launched IKONOS and QuickBird. The latter two were developed to provide high-resolution imagery for both civil and government use. Many (>30) new remote sensing satellite systems are now operational in addition to 12 further planned launches within the next year [20], which boast even higher image resolution and positional accuracy. IKONOS provides spatial resolution of up to $0.8 \mathrm{~m}$ panchromatic ground sample distance (GSD) and $4 \mathrm{~m}$ multispectral GSD, whereas QuickBird's resolution is sharper at $0.6 \mathrm{~m}$ and $2.4 \mathrm{~m}$ [20]. Several agencies sell these high resolution images; however, they are often too expensive for the average user, as a minimum purchase area applies.

Aerial photographs provide a useful alternative to satellite imagery, because they have the advantage of being acquired at closer-range than satellites, and consequently provide higher scale and detail/resolution. These two attributes are necessary to assist enhanced waypoint identification. For example, an aerial photo taken at $300 \mathrm{~m}$ above ground level with the "normal" $150 \mathrm{~mm}$ focal length lens has a resolution on the ground of $0.08 \mathrm{~m}$ per pixel [21], which is more precise than both IKONOS and QuickBird. It is worth mentioning that another low cost approach for acquiring aerial imagery is a system for remote sensing in times of disaster [22], which could be used for waypoint based navigation. In this, a mechatronic kite equipped with a teleoperated camera, video transmitter, battery, remote controlled receiver and two servos (for pan and tilt) have been used for live data capture. The advantages are that it can be deployed rapidly, is lightweight and can quickly obtain images. Finally, another method of 
capturing aerial images includes using an Unmanned Aerial Vehicle that obtains aerial LADAR data [8]. Irrespective of the image used, post-image processing is required for georeferencing.

\section{B. Georeferencing}

This is the process in which the image is related to a suitable ground coordinate system. Since the earth is not a perfect sphere, setting these factors to a fixed universal mathematical index such as the widely used World Geodetic System 1984 (WGS84), could lead to inaccuracies of several meters, depending on the geographical location of the image in the global frame [23]. This leads to two concepts: Map Datum and Map Projection [23]. It is important to set the aerial images to the datum and projection used to represent the country in which the image was taken. In the UK, for example, the map projection used is known as the Transverse Mercator (TM) and the Map Datum as the Ordnance Survey Great Britain 1936, which is based on a geographic representation known as the Airy 1830 ellipsoid. Direct transformations between various map datums (e.g. OSGB36 to WGS84) can be achieved using for example the Helmert Transformation. Unfortunately such transformations are meaningless at the local scale. In the UK for instance, small scale inaccuracies arising from the 1936 re-triangulation lead to significant

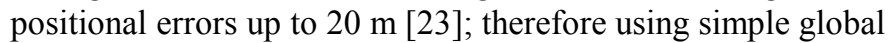
transformations and published constants is not advisable. It is important to ensure that a consistent underlying coordinate system for the aerial image being used, and that the GPS positional output matches its corresponding location on the image.

\section{Photogrammetry}

The science developed to relate measurements of imagery to a ground coordinate system is known as photogrammetry [24], the impetus for development being primarily the production of the World's National Mapping series [25]. There are two types of distortion inherent in any aerial or satellite image, which prevent direct correspondence between the 2D image and a 3D ground coordinate system: tilt and relief distortion. Distortions created by the light rays leaving the object, passing through the lens center, before creating an image point in the focal plane of the camera are modeled explicitly using the collinearity equations [24],[25]. These equations model completely distortions due to non-verticality of the sensor. A distortion is also introduced into the image if the terrain is non-planar. Such "relief displacements" are related to the flying height and focal length of the sensor and can be highly significant for aerial photography. Only a true "orthorectification" procedure implementing the collinearity equations removes the distortions due to both relief and tilt displacement. Unfortunately there are a range of aerial image products marketed which have not been generated using the required rigorous mathematical procedures. Although such "map accurate" products are fit for many purposes/applications, they should always be used with caution, particularly when used in conjunction with GPS.
The process of orthorectification can introduce discrepancies if the Digital Elevation Model is inaccurate. Therefore, it is important to consider such uncertainties when judging the inaccuracy of the waypoint selected from an aerial image.

\section{RoBotic SYSTEM}

\section{A. Global Positioning System}

Since the GPS was selected as a stand-alone localization sensor, this section is dedicated to providing a brief description of it's capabilities. Currently there exist two active satellite positioning systems, the US GPS and the former USSR GLONASS [26]; however, the first satellite of the "Galileo" European positioning system has been launched.

A solution to inaccuracies stemming from atmospheric conditions, orbit instability, and disturbances in the satellite constellation were adjusted by accurately georeferenced ground stations, which acted as beacons and transmitted corrected GPS signals [27]. This is known as differential GPS (DGPS). However, the accuracy of the corrected signals degrades as the distance from these stations increases, and subscription can be costly. This ground-beacon distance problem has been handled by the introduction of geostationary satellites that transmit differentially corrected signals. In the US this system is known as Wide Area Augmented System (WAAS) and in Europe as the European Geostationary Navigation Overlay Service (EGNOS) and can provide civilians with positioning accuracies to within $3 \mathrm{~m}$. The GPS has become a topical subject amongst researchers; however, rarely is it used independently for localization.

\section{B. Kinematics and Controller}

Reference [28] presents the controller used for a simulation of a synchronous drive robot, which is to be used to verify the efficiency of the control system in reaching waypoints using a simulated positional output from the GPS. One general kinematic model for a synchronous drive robot can be found in work done in [29]. However, given that a convenient model to work with in the Simulink ${ }^{\circledR}$ environment is not readily available, a detailed step-by-step derivation is presented. The following assumptions are made: the robot has synchronous wheel rotation, a symmetric wheel configuration (square wheel configuration), homogeneous wheel radii, no lateral or longitudinal wheel slip, no wheel misalignments, no pressure differences in tires, no dynamic modeling, and moves along a 2-D (horizontal) plane.

This simplifies the kinematic model to the basic constraints acting on the robot. A schematic is used for the derivation (Fig. 2). Table I summarises the variables.

The main feature of a robot with such a mechanism is that all the wheels can rotate simultaneously $360^{\circ}$ continuously and unhindered at the same angular velocity, direction and translational velocity.

For that reason the instantaneous centre of curvature (rotation) (ICC or ICR) is at infinity. 
TABLE I

SYMBOLS, DESCRIPTION AND UNITS FOR SPIDER's KINEMATIC MODEL

\begin{tabular}{ccc}
\hline \hline Symbol & Description & Units \\
\hline$\theta$ & Steering angle & Radians \\
$\dot{\theta}$ & Steering velocity & Radians/Second \\
$\alpha$ & Angle of rotation of & Radians \\
& Spider frame to global & \\
& frame & \\
$d,-d$ & Robot dimension & Meters \\
& along $X_{\text {Spider }}$ axis & \\
$b,-b$ & Robot dimension & Meters \\
& along $Y_{\text {Spider }}$ axis & Radians \\
$\phi$ & Wheel angle & Radians/Second \\
$\dot{\phi}$ & Wheel angular velocity & Meters/Second \\
$v$ & Robot linear velocity & (Meters, Meters, Radians) \\
$\xi$ & Robot posture vector & Radians/Second) \\
$\dot{\xi}$ & Robot velocity vector & (Meters/Second, Meters/Second, \\
& in global frame & Rame \\
\hline \hline
\end{tabular}

This leads to the fact that the robot's frame will remain constant by an angle $(\alpha)$ to the global reference frame, unless wheel slipping or other unforeseen external dynamic factors occur.

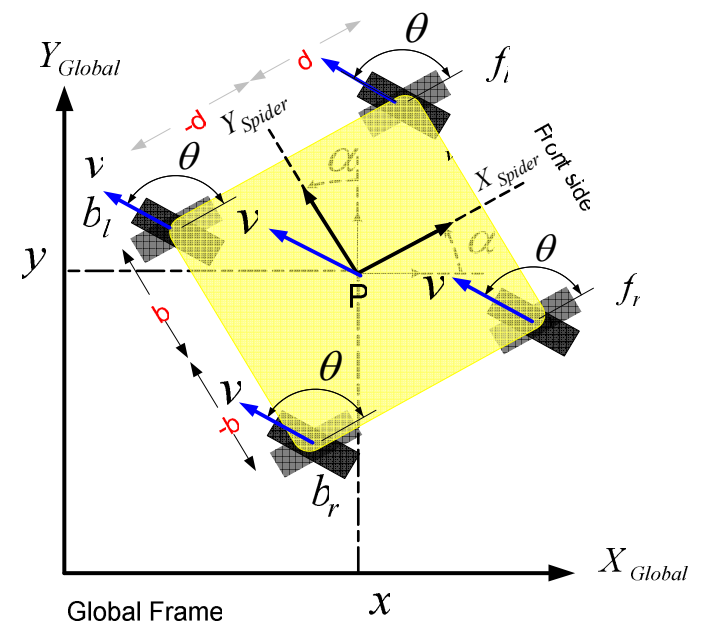

Fig. 2. Kinematic model of the four-wheeled synchronous drive robot.

The robot posture ( $\xi_{\text {Global }}$ ) can be defined by the following vector representation:

$$
\xi_{\text {Global }}=\left[\begin{array}{lll}
x & y & \alpha
\end{array}\right]^{T}
$$

Since the global reference frame and the robot frame are not aligned, it is necessary to map the motion of the global frame to that of the robot. To achieve this, an orthogonal rotation matrix $(R(\alpha))$ is needed:

$$
R(\alpha)=\left[\begin{array}{ccc}
\cos \alpha & \sin \alpha & 0 \\
-\sin \alpha & \cos \alpha & 0 \\
0 & 0 & 1
\end{array}\right]
$$

The calculation is denoted by:

$$
\xi_{\text {Spider }}=R(\alpha) \xi_{\text {Global }}
$$

The next stage is to calculate the wheel's kinematic constraints, which determine the motion constraints of each wheel. Since this is a synchronous mechanism, the calculation of one wheel is sufficient. For this, both constraints orthogonal to and along the wheel plane need to be determined. Please refer to Fig. 3.

In order to compute the correct constraints, it is vital to determine the type of wheel being used. For this robot it belongs to the class of steered standard wheels. Reference [30] provides further explanation. The resolved equations are:

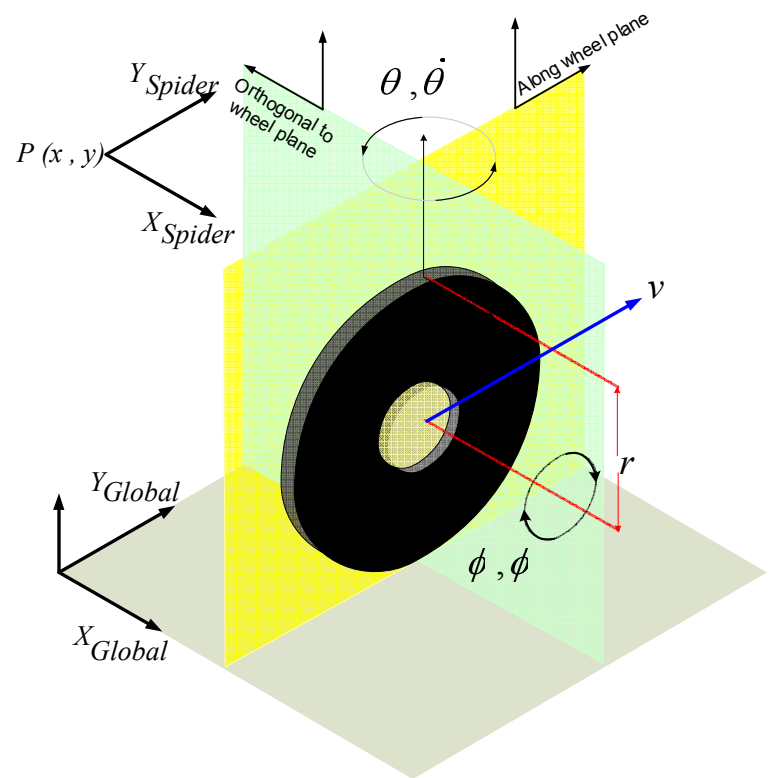

Fig. 3. Wheel's kinematic constraints frames: orthogonal and along wheel plane.

Along the wheel plane:

$$
\begin{gathered}
{\left[\begin{array}{cc}
\cos \left(\theta_{i}\right) & \sin \left(\theta_{i}\right) \quad d_{i} \sin \left(\theta_{i}\right)-b_{i} \cos \left(\theta_{i}\right)
\end{array}\right]} \\
\cdot R(\alpha) \dot{\xi}_{\text {Global }}-r \dot{\phi}_{i}=0
\end{gathered}
$$

Orthogonal to the wheel plane:

$$
\begin{gathered}
{\left[\begin{array}{ll}
-\sin \left(\theta_{i}\right) & \cos \left(\theta_{i}\right) \quad d_{i} \cos \left(\theta_{i}\right)+b_{i} \sin \left(\theta_{i}\right)
\end{array}\right]} \\
\cdot R(\alpha) \dot{\xi}_{\text {Global }}=0
\end{gathered}
$$

Where $\dot{\xi}_{\text {Global }}=\left[\begin{array}{lll}\dot{x} & \dot{y} & \dot{\alpha}\end{array}\right]^{T}$ is the robot's posture velocity vector, $\theta_{i}$ is the steering angle at a certain instant in time, and $d_{i}, b_{i}$ are the positions of the wheels with respect to point $P$ along the robot's frame. Given that the Spider has a symmetric four wheel configuration $(d=b)$, then:

$$
\begin{gathered}
d_{f l}=d_{f r}=b_{b l}=b_{f l}=d \\
d_{b r}=d_{b l}=b_{b r}=b_{f r}=-d
\end{gathered}
$$

Therefore, (4) and (5) can be modified with the dimensions presented in (6) to obtain the full kinematic wheel constraints acting on the wheel frame. Hence, along the wheel plane: 


$$
\begin{aligned}
& b_{r:} \quad\left[\begin{array}{lll}
\cos \left(\theta_{b r}\right) & \sin \left(\theta_{b r}\right) & -d \sin \left(\theta_{b r}\right)+d \cos \left(\theta_{b r}\right)
\end{array}\right] \\
& \cdot R(\alpha) \dot{\xi}_{\text {Global }}-r \dot{\phi}_{b r}=0 \\
& b_{l}:\left[\begin{array}{lll}
\cos \left(\theta_{b l}\right) & \sin \left(\theta_{b l}\right) & -d \sin \left(\theta_{b l}\right)-d \cos \left(\theta_{b l}\right)
\end{array}\right] \\
& \cdot R(\alpha) \dot{\xi}_{G l o b a l}-r \dot{\phi}_{b l}=0 \\
& f_{r:}\left[\begin{array}{lll}
\cos \left(\theta_{f r}\right) & \sin \left(\theta_{f r}\right) & d \sin \left(\theta_{f r}\right)+d \cos \left(\theta_{f r}\right)
\end{array}\right] \\
& \cdot R(\alpha) \dot{\xi}_{G l o b a l}-r \dot{\phi}_{f r}=0 \\
& f_{l}:\left[\begin{array}{lll}
\cos \left(\theta_{f l}\right) & \sin \left(\theta_{f l}\right) & d \sin \left(\theta_{f l}\right)-d \cos \left(\theta_{f l}\right)
\end{array}\right] \\
& \cdot R(\alpha) \dot{\xi}_{G l o b a l}-r \dot{\phi}_{f l}=0
\end{aligned}
$$

Orthogonal to wheel plane:

$$
\begin{gathered}
\left.b_{r}: \quad \begin{array}{cc}
-\sin \left(\theta_{b r}\right) & \cos \left(\theta_{b r}\right) \quad-d \cos \left(\theta_{b r}\right)-d \sin \left(\theta_{b r}\right)
\end{array}\right] \\
\cdot R(\alpha) \dot{\xi}_{\text {Global }}=0
\end{gathered}
$$$$
b_{l}:\left[\begin{array}{lll}
-\sin \left(\theta_{b l}\right) & \cos \left(\theta_{b l}\right) & -d \cos \left(\theta_{b l}\right)+d \sin \left(\theta_{b l}\right)
\end{array}\right]
$$$$
\cdot R(\alpha) \dot{\xi}_{\text {Global }}=0
$$

$$
\begin{gathered}
f_{r}:\left[\begin{array}{ccc}
-\sin \left(\theta_{f r}\right) & \cos \left(\theta_{f r}\right) & d \cos \left(\theta_{f r}\right)-d \sin \left(\theta_{f r}\right)
\end{array}\right] \\
\cdot R(\alpha) \dot{\xi}_{\text {Global }}=0
\end{gathered}
$$

$$
\begin{aligned}
& f_{l}:\left[\begin{array}{lll}
-\sin \left(\theta_{f l}\right) & \cos \left(\theta_{f l}\right) & d \cos \left(\theta_{f l}\right)+d \sin \left(\theta_{f l}\right)
\end{array}\right] \\
& \cdot R(\alpha) \dot{\xi}_{\text {Global }}=0
\end{aligned}
$$

Followed by this the kinematic constraints need to be expressed in the Matrix form $A(q) \dot{q}=0$. In order to obtain the state space representation of the robot, it is important to determine the null space of $A(q)$ for $v=r . \dot{\phi}$ and include the steering velocity $\dot{\theta}$. Hence the representation in the form of $\dot{q}=S_{q} u$ :

$$
\left[\begin{array}{c}
\dot{x} \\
\dot{y} \\
\dot{\alpha} \\
\dot{\theta}_{b r} \\
\dot{\theta}_{b l} \\
\dot{\theta}_{f r} \\
\dot{\theta}_{f l} \\
\dot{\phi}_{b r} \\
\dot{\phi}_{b l} \\
\dot{\phi}_{f r} \\
\dot{\phi}_{f l}
\end{array}\right]=\left[\begin{array}{cc}
\cos (\theta+\alpha) & 0 \\
\sin (\theta+\alpha) & 0 \\
0 & 0 \\
0 & 1 \\
0 & 1 \\
0 & 1 \\
0 & 1 \\
1 / r & 0 \\
1 / r & 0 \\
1 / r & 0 \\
1 / r & 0
\end{array}\right]\left[\begin{array}{c}
v \\
\dot{\theta}
\end{array}\right]
$$

Since $\dot{\theta}_{i}=\dot{\theta} \forall i, \dot{\phi}_{i}=\dot{\phi} \forall i$ and $v=r . \dot{\phi}$, (9) can be reduced to:

$$
\left[\begin{array}{c}
\dot{x} \\
\dot{y} \\
\dot{\alpha} \\
\dot{\theta} \\
\dot{\phi}
\end{array}\right]=\left[\begin{array}{cc}
r \cdot \cos (\theta+\alpha) & 0 \\
r \cdot \sin (\theta+\alpha) & 0 \\
0 & 0 \\
0 & 1 \\
1 & 0
\end{array}\right]\left[\begin{array}{c}
\dot{\phi} \\
\dot{\theta}
\end{array}\right]
$$

Referring back to (10), it can be seen that five factors are needed to determine the robot's velocity components in the $x-y$ plane (forward kinematics), where the robot's steering velocity $\dot{\alpha}$ is zero (i.e., the orientation of the robot's platform $\alpha$ never changes under the above mentioned conditions).

\section{EXPERIMENTAL RESULTS}

In order to conduct the set of tests presented in this paper the following were used: 1) a $0.18 \mathrm{~m} /$ pixel resolution aerial image of the Holywell car park at Loughborough University, orthorectified into Ordnance Survey coordinates (British National Grid); 2) two Leica System 500 receivers for precise differential point positioning using static data post-processing (horizontal accuracy $5 \mathrm{~mm}+1 \mathrm{ppm}$, vertical accuracy $10 \mathrm{~mm}+$ $1 \mathrm{ppm}$ ); 3) Garmin 18 5Hz GPS unit; 4) Matlab Simulink model for a synchronous drive robot; 5) Erdas Imagine 9.0 by Leica Geosystems and 6) freely available GPS planning software (Trimble planning software).

\section{A. Aerial Image and Waypoint Accuracy}

In order to show the nature of disparities between a georeferenced aerial image and waypoints, two tests were performed. In the first one, the Leica System was used to collect 54 points using a survey style "stop-and-go" approach, in an attempt to measure points covering the majority of the parking lot. These points are superimposed on the aerial image (Fig. 4).

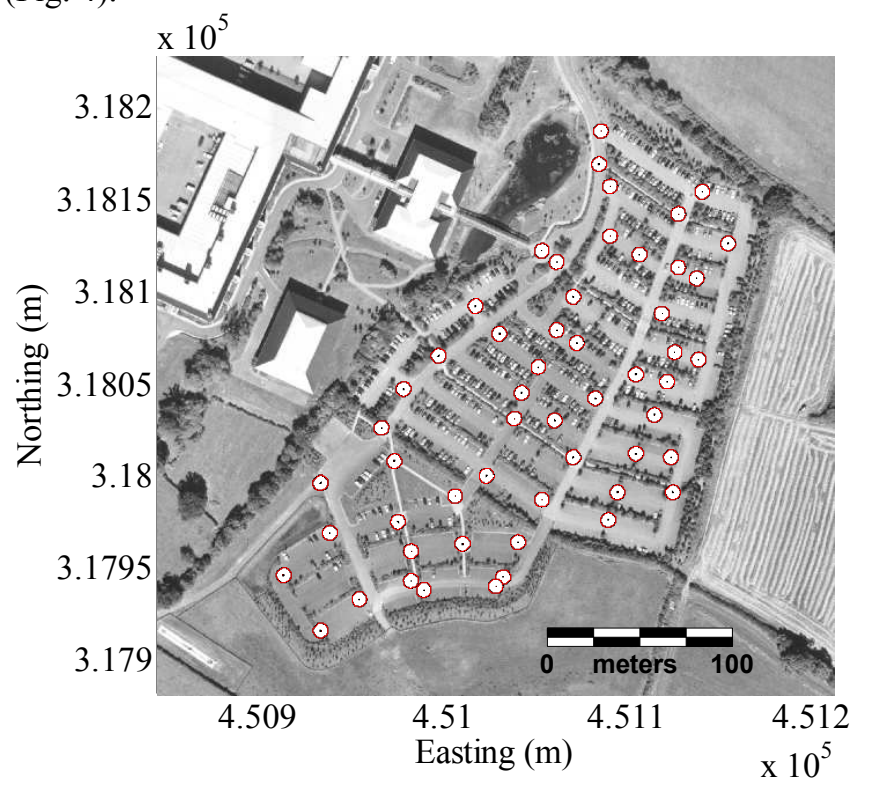

Fig. 4. 55 accurately surveyed points using static post data processing. 
Clearly recognizable and identifiable landmarks on the image (marked as waypoints) were chosen as points to be surveyed by the high precision GPS on their corresponding points in the car park. It can be clearly seen in Fig. 5 that many of the waypoints selected do not match their corresponding surveyed points exactly. It was determined that, for the clearly recognizable points $(37 / 54)$, the surveyed points had an average $0.37 \mathrm{~m} \mathrm{~N} / \mathrm{E}$ shift from the user selected waypoints (varying from $0.087 \mathrm{~m}$ to $0.732 \mathrm{~m}$ ) (see Fig. $5(\mathrm{a}, \mathrm{b}$ ) for a waypoint comparison). On the other hand, for the entire data set $(54 / 54)$, an average $0.446 \mathrm{~m} \mathrm{~N} / \mathrm{E}$ shift from the user selected waypoints was obtained (varying from $0.087 \mathrm{~m}$ to $2.085 \mathrm{~m}$ ). Such differences can be accounted by the presence of variability and bias error. The bias error arises from small inaccuracies involved in the measurement process, most significant being slightly varying parameters in the processing between the established photo-control and established check points. There was also a small and systematic height bias in the extracted DEM, which causes a systematic shift in the position of the pixels comprising the orthorectified image. The variability usually relates to natural human induced variation; waypoints selected from an image by one person may differ from a set collected by another. This is represented by the range, or standard deviation.

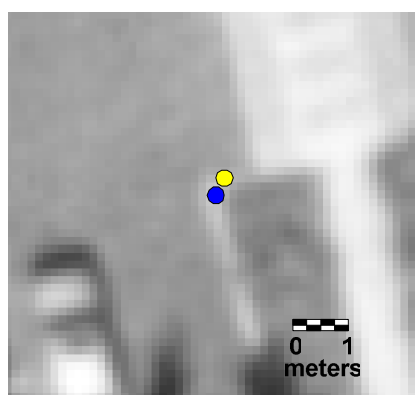

(a)

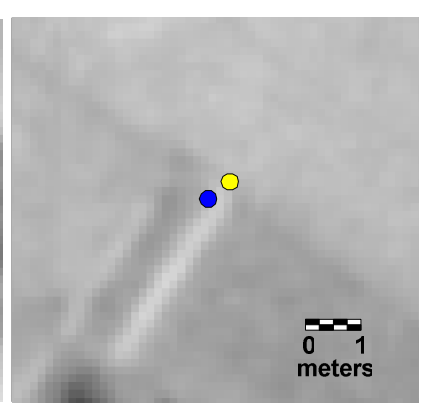

(b)
Fig. 5. a) Shows a shift between the dark colored user selected point and the light colored surveyed point; b) shows that the user selected waypoint is in fact in a better "visual" position than the actual measured waypoint on that site.

Given the variation of the shift throughout the image, it is evident that it is not entirely possible to match an image waypoint to the actual location in the car park. Therefore it is important to define a proximity error around each waypoint. This proximity error, however, is left up to the user to define since it should be based on the image resolution, the image positional inconsistencies due to orthorectification, and human error concerning waypoint selection. It is possible to recalibrate the image to the standard needed; however, this would be daunting task for the average user, and might be beyond the accuracy needed.

This leads to the next set of experimental results that demonstrate the importance of adjusting the GPS receivers' positional shift, to improve the spatial match between the GPS data and the orthorectified image.

\section{B. GPS Positional Correction}

WGS84 is the default coordinate system adopted by the GPS receiver. Any other coordinate system selected would be based on a mathematical transformation from the default - which as previously stated yields erroneous results (see Georeferencing). Because the GPS showed positional variation for a single spot from one day to the next, irrespective of the coordinate system chosen, it was determined that adopting a mathematical spatial shift would inevitably provide significantly improved positional accuracy. This would overcome some of these computational errors obtained due to the receiver's internal Molodensky coordinate system transformation [31]. This would provide "corrected" (or tuned) positional data, suitable for a certain time period and geographic location. The approach adopted showed positional stability for approximately 1.5 hours of testing. Since it was also determined that the OSGB36 coordinate system was spatially not as close to the position being measured as anticipated, parameters local to the test area were obtained to improve the overall spatial position. This would therefore reduce the amount of mathematical compensation needed. The constants used for the "User Defined Settings" were: inverse flattening factor $(D f): 299.3249646$; the semimajor axis, equatorial radius ( $D a)$ : 6377563.396; positional shift along $\mathrm{x}$ axis $(d x)$ : 371 ; positional shift along y axis $(d y)$ : -112 ; and the positional shift along $\mathrm{z}$ axis $(d z): 434$. This is based on the Airy 1830 ellipsoid.

The GPS positional tuning was conducted using the following method: one point in a relatively open area was precisely surveyed. The Garmin GPS was then placed on the same location, at a height equivalent to the robot's GPS height of $1.5 \mathrm{~m}$, to determine an average value over a 15 minute sample time. The data was then converted to Eastings/Northings, and compared to its corresponding surveyed point. The positional shift was then used to compensate for the positional output from the GPS during forthcoming tests.

One test, conducted on the car park premises, shows the result of the path data before and after this GPS positional correction approach. The GPS unit was attached to a trolley and driven around a designated marked line in the road's center. Fig. 6 shows the effect of this positional correction. The tests were conducted for 55 minutes for a total traveled distance of $1.3 \mathrm{~km}$ (each turn $420.8 \mathrm{~m}$ ).

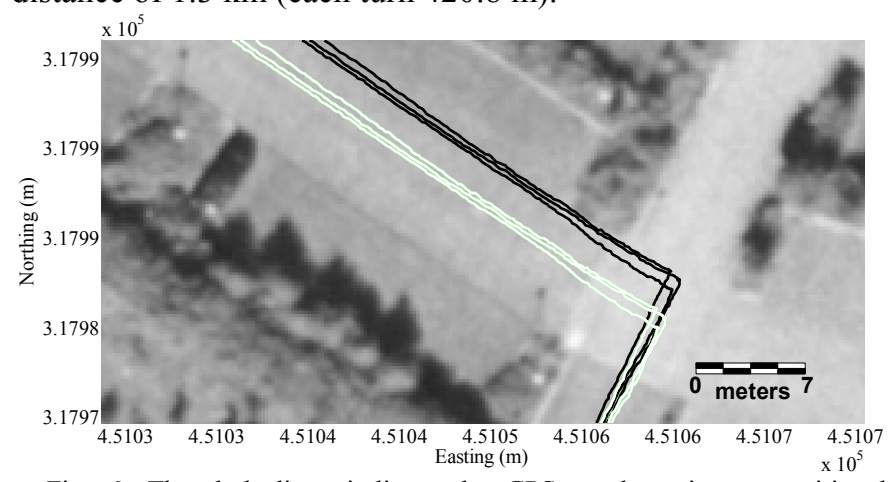

Fig. 6. The dark lines indicate the GPS results prior to positional correction, and the light lines indicate the post positional correction. 
In order to test the repeatability, the error of the GPS and furthermore the number of waypoints hit, a mock test was created. A series of waypoints, seen in Fig. 7, were created and the GPS-mounted trolley was driven through them for 17 runs $(\sim 1.43 \mathrm{~km})$. In order to ensure optimal results, the Trimble planning software was used to determine the most suitable time for testing.

An open space area was used to ensure an unobstructed sky view, and no vehicles were present. For the majority of the time there were 10 satellites in view with a horizontal dilution of precision (Hdop) ranging from 0.9 to 1.1 , occasionally reaching 1.3 .

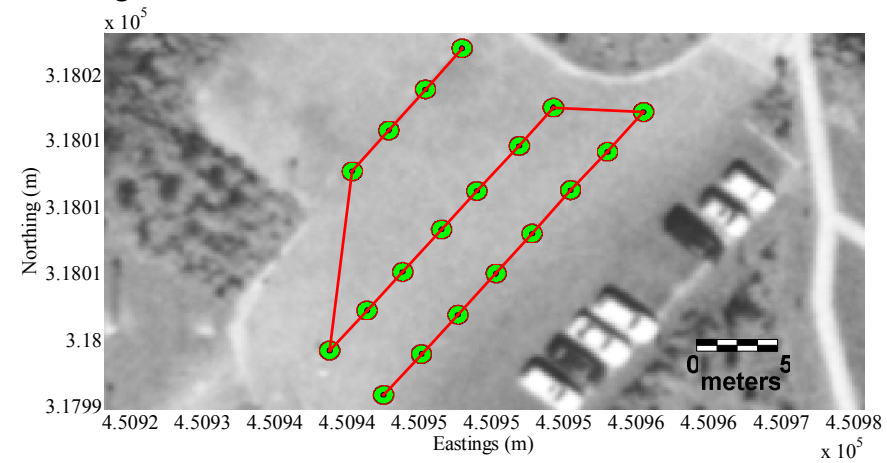

Fig. 7. 19 waypoints used for testing GPS positional correction approach, repeatability and accuracy.

The results for varying the waypoint radius, for both postand pre-calibration can be seen in Fig. 8. Followed by the testing, the GPS calibration was checked once more to determine an easting shift of $0.07 \mathrm{~m}$ and approximately $0.3 \mathrm{~m}$ in the northing. The percentage of waypoints hit may vary from one day to the other, and therefore, depending on the accuracy required, the proximity error (radius) can be adjusted. Present GPS positioning data shows improved positional accuracy for mobile robot navigation compared to results prior to the deployment of the geostationary satellites (EGNOS/WAAS) [32].

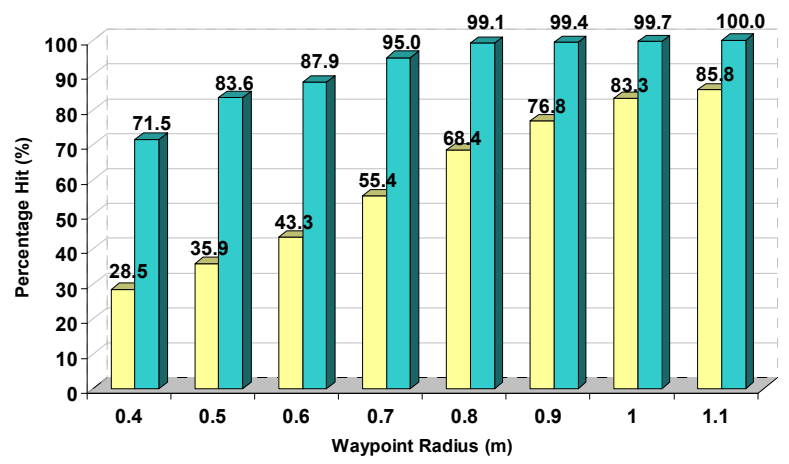

Fig. 8. The percentage of waypoints hit for 17 runs before and after positional correction. $\square$ - before correction, $\square$ - after correction.

\section{Simulation Results - Final - entire system}

To test the effectiveness of the control system in reaching a waypoint with proximity error, the following simulation is presented. A challenge with such a simulation is the ability to mimic the positional accuracy of the GPS. Therefore, the GPS results from the field work were used to obtain an estimate of this positional behavior after mathematical compensation. It was determined that by using a continuous random number generator function that produces an overall uniform normal distribution in addition to a random positional shift anywhere within a user selected radius (that is set once at the beginning of each simulation) yielded results similar to the real GPS. With the addition of this simulated positional response, a level of uncertainty and unpredictability is added making the simulation more realistic. For consistency the robot's simulated linear velocity was fixed at $5 \mathrm{~km} / \mathrm{h}$ and its angular velocity at 0.76 radians/s ( 1 revolution in $\sim 9$ seconds). The robot has a square configuration of $1.3 \times 1.3 \mathrm{~m}$. The proximity error of the GPS was set to within $1.6 \mathrm{~m}$ and the individual positional data to within $0.2 \mathrm{~m}$.

The results yielded a waypoint hit of $94.73 \%$ for 18 test runs. A hit was considered only when the robot's center passed through the proximity error of the waypoint. The efficiency of that controller however also depends on the speed and the angular velocity of the robot. A higher hit count was achieved at a lower linear velocity. The majority of the misses occurred during turning maneuvers (due to overshoot). A future modified version of the controller would be one that would vary its translational and angular velocity during turning to ensure that the waypoint is reached. Fig. 9 is the view of one of the simulations.

This therefore indicates that the simulation can be used as a reasonable benchmark for testing various control systems prior to deployment. If this controller is to be placed into the working robot, similar results are expected. Hence the global task of using waypoints selected from aerial images, with corrected positional data from a GPS and suitable controller could achieve promising accuracies depending on the application needed.

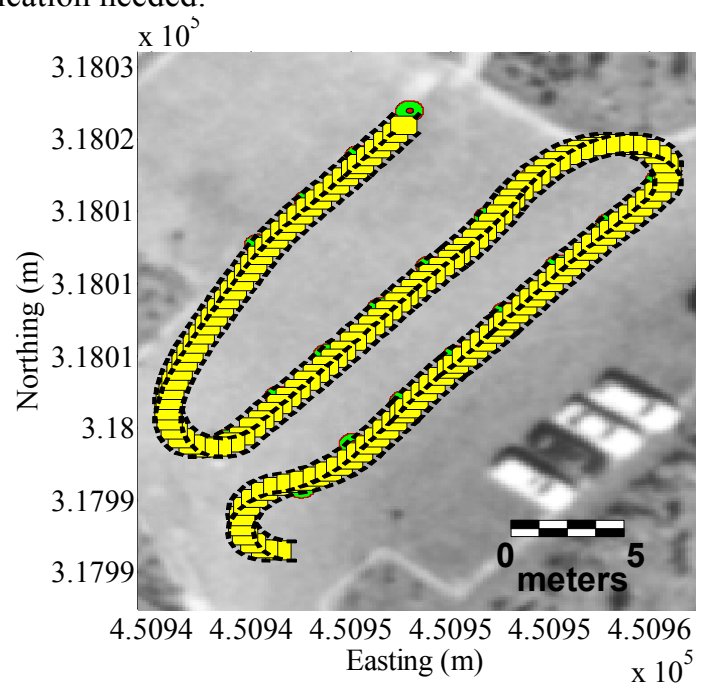

Fig. 9. The robot passing through the waypoints in a simulated GPS environment.

\section{ACKNOWLEDGMENT}

The authors would like to thank the department of Civil Engineering at Loughborough University, for providing the use of their survey grade equipment, and the technicians Dave Liddell and Terry Harrison, without whom this work would not have been possible. 


\section{REFERENCES}

[1] S. Shair, R. M. Parkin, and M. R. Jackson, "A robust mobile platform for autonomous and teleoperative navigstion" in Proc. $10^{\text {th }}$ International Conference on Mechatronics Technology, México, 2006, pp. 1-6.

[2] J. Meguro et al., "Creating spatial temporal database by autonomous mobile surveillance system (a study of mobile robot surveillance system using spatial temporal GIS Part 1)," in Proc. of the 2005 IEEE International Workshop on Safety, Security and Rescue Robotics, Kobe, Japan, 2005.

[3] D. Silver, B. Sofman, N. Vandapel, J. A. Bagnell, and A. Stentz, "Experimental analysis of overhead data processing to support long range navigation," in Proc. of the 2006 IEEE/RSJ International Conference on Intelligent Robots and Systems, Beijing, China, 2006.

[4] P. Sim, V. Sacco, and G. S. Virk, "Robot navigation in volcanic environments," in Proc. of the 2003 IEEE Conference on Intelligent Transportation Systems, Shanghai, China, 2003

[5] S. Zein-Sabatto, O. Taiwo, and P. Koseeyaorn. "Integrated simulation software for outdoor robots planning and coordination," in Proceedings of the 2004 IEEE Southeast Conference, Greensboro, North Carolina, 2004 .

[6] A. Howard, H. Seraji, and B. Werger, "Global and regional path planners for integrated planning and navigation," in Journal of Robotic Systems, vol. 22, no. 12, pp. 767-778, 2005.

[7] G. Muscato et al., "ROBOVOLC: A robot for volcano exploration result of first test campaign," Industrial Robot, vol. 30, no. 3, pp. 231-242, 2003.

[8] N. Vandapel, R. Donamukkala, and M. Hebert, "Experimental results in using aerial LADAR data for mobile robot navigation," presented at the 4th International Conference on Field and Service Robotics, Fuji, Japan, 2003.

[9] B. Sofman, E. Lin, J. Bagnell, N. Vandapel, and A. Stentz, "Improving robot navigation through self-supervised online learning," in Proceedings of Robotics: Science and Systems Conference, Philadelphia, Pennsylvania, 2006.

[10] B. Sethuramasamyraja, "GPS based waypoint navigation for an autonomous guided vehicle - Bearcat III," M.S. thesis, Dept. of Mechanical, Industrial and Nuclear Engineering, Univ. of Cincinnati, 2003.

[11] M. Wuersch and D. Caduff, "Refined route instructions using topological stages of closeness," in Lecture Notes in Computer Science, Berlin/Heidelberg, Germany, SpringerLink, 2005.

[12] V. Ashkenazi, D. Park, and M. Dumville, "Robot positioning and the global navigation satellite system," Industrial Robot, vol. 27, no. 6, pp. 419-426, 2000.

[13] S. Cameron, "Obstacle avoidance and path planning," Industrial Robot, vol. 21, no. 5, pp. 0143-991X, 1994

[14] H. Durrant-Whyte, "Autonomous land vehicles," in Proc. of the Institution of Mechanical Engineers, Part I: Journal of Systems and Control Engineering, vol. 219, no. 1, 2005, pp. 77-98.

[15] M. E. Holden, "Low-cost autonomous vehicles using just GPS Waypoint navigation," in Proc. of the 2004 ASEE Conference, Salt Lake City, Utah.

[16] T. W. Vaneck, "Fuzzy guidance controller for an autonomous boat," IEEE Control Systems Magazine, vol. 17, no. 2, pp. 43-51, 1997.

[17] M. Kumon, Y. Udo, H. Michihira, M. Nagata, I. Mizumoto, and Z. Iwai, "Autopilot system for kiteplane," IEEE/ASME Transactions on Mechatronics, vol. 11, no. 5, pp. 615-624, Oct. 2006.

[18] M. H. Bruch, G. A. Gilbreath, J.W. Muelhauser, and J. Q. Lum, "Accurate waypoint navigation using non-differential GPS," presented at AUVSI Unmanned Systems 2002, Lake Buena Vista, FL, July 9-11.

[19] J. Feddema, C. Lewis, and P. Klarer. "Control of multiple robotic sentry vehicles," In Proc. of SPIE: The International Society for Optical Engineering, Orlando, FL, 1999.

[20] "Earth observation sensors: higher resolutions and shorter revisit times,"in Engineering Surveying Showcase 2007, no. 2, 2007, pp. 1925.

[21] D. T. Booth, S. E. Cox, and R. D. Berryman, "Precision measurements from very-large scale aerial digital imagery," Environmental Monitoring and Assessment, vol. 112, no. 1-3, pp. 293-307, 2006.
[22] P. Y. Oh and W. E. Green, "Mechatronic kite and camera rig to rapidly acquire, process, and distribute aerial images," IEEE/ASME Transactions on Mechatronics, vol. 9, no. 4, pp. 671 - 678, 2004.

[23] A guide to coordinate systems in Great Britain, vol. 1.7, Ordnance Survey, Southampton, UK, Oct. 2007.

[24] J. Fryer, H. Mitchell and J. Chandler, Applications of 3D measurement from Images. Caithness, Scotland: Whittles Publishing, 2007, pp. 304.

[25] P.R. Wolf, Elements of Photogrammetry. Singapore: McGraw-Hill Companies, 1983, pp. 628.

[26] A. Michalski and J. Czajewski, "The accuracy of the global positioning systems," IEEE Instrumentation and Measurement Magazine, vol. 7, no. 1, pp. 56-60, 2004.

[27] C. Satirapod, I. Trisirisatayawong, and P. Homniam, "Establishing ground control points for high-resolution satellite imagery using GPS precise point positioning," in Proc. of the IEEE International Geoscience and Remote Sensing Symposium, Toulouse, France, 2003.

[28] V. J González-Villela, R. M. Parkin, M. Lopez-Parra, J. M. DoradorGonzález and L. Guadarrama, "A wheeled mobile robot with obstacle avoidance capability," Ingeniería Mecánica Tecnología y Desarrollo. Revista de la Sociedad Mexicana de Ingeniería Mecánica (SOMIM), vol. 1, no. 5, pp. 159-166, 2004.

[29] D. Fox, W. Burgard, and S. Thrun, "The dynamic window approach to collision avoidance," IEEE Robotics and Automation Magazine, vol. 4, no. 1 , pp. 23-33, 1997

[30] R. Siegwart and I. R. Nourbakhsh, Introduction to Autonomous Mobile Robots. Cambridge, MA: MIT Press, 2004.

[31] D. DePriest, A GPS User Manual: Working with Garmin Receivers. Fairfield, CA: $1^{\text {st }}$ Books, 2003, ch. 5.

[32] S. Panzieri, F. Pascucci, and G. Ulivi, "An outdoor navigation system using GPS and inertial platform," IEEE/ASME Transactions on Mechatronics, vol. 7, no. 2, pp. 134-142, June 2002.

S. Shair obtained his B.Sc. in mechanical engineering from Queen's University, Kingston, Ontario, Canada in 2005. He is currently pursuing a Ph.D. degree at the Wolfson School of Mechanical and Manufacturing Engineering, Loughborough University, Loughborough, UK.

J.H. Chandler obtained his B.Sc in land surveying and geography in 1983, graduating from Newcastle University, Newcastle, UK. He achieved his $\mathrm{PhD}$ at City University, London, UK in 1989 and is currently a senior lecturer in the Department of Civil and Building Engineering, Loughborough University, Loughborough, UK.

V.J. Gonzalez-Villela obtained his B.Eng. in mechanical and electrical Engineering in 1987 and M.Eng. in electrical engineering in 1993 both from the National Autonomous University of Mexico (UNAM), Mexico City, Mexico. He achieved his PhD at Loughborough University, UK in 2006. He is currently Professor in the Department of Mechatronics Engineering at UNAM.

R.M. Parkin gained a B.Sc in engineering science from Leicester University and a PhD from Leicester Polytechnic, Leicester, UK in 1976 and 1980, respectively. He was a founder member of the UK Mechatronics Forum and is a Professor of Mechatronics and now Head of the Wolfson School of Mechanical \& Manufacturing Engineering at Loughborough University, Loughborough, UK.

M.R. Jackson obtained his B.Sc (Hons) in mechanical engineering from Salford University, Salford, UK, in 1975. He obtained his PhD through the CNAA and Leicester Polytechnic, Leicester, UK, in 1986. He obtained his M.Sc in flexible manufacturing systems \& robotics at Loughborough University, Loughborough, UK, in 1987. He is currently Professor of Machine Systems at the Wolfson School of Mechanical \& Manufacturing Engineering at Loughborough University. 